\title{
THE VARIATION OF SINGULAR CYCLES IN AN ALGEBRAIC FAMILY OF MORPHISMS
}

\author{
BY \\ JOEL ROBERTS $\left({ }^{1}\right)$
}

\begin{abstract}
Let $g: V^{r} \rightarrow W^{m}(m \geqq r)$ be a morphism of nonsingular varieties over an algebraically closed field. Under certain conditions, one can define a cycle $S_{i}$ on $V$ with Supp $\left(S_{i}\right)=\left\{x \mid \operatorname{dim}_{k(x)}\left(\Omega_{X / Y}^{1}\right)(x) \geqq i\right\}$.

The multiplicity of a component of $S_{i}$ can be computed directly from local equations for $g$. If $V^{r} \subset \boldsymbol{P}^{n}$, and if $g: V \rightarrow \boldsymbol{P}^{m}$ is induced by projection from a suitable linear subspace of $\boldsymbol{P}^{n}$, then $S_{1}$ is $c_{m-r+1}(N \otimes \mathcal{C}(-1))$, up to rational equivalence, where $N$ is the normal bundle of $V$ in $P^{n}$.

(2) Let $f: X \rightarrow S$ be a smooth projective morphism of noetherian schemes, where $S$ is connected, and the fibres of $f$ are absolutely irreducible $r$-dimensional varieties. For a geometric point $\eta$ : Spec $(k) \rightarrow S$, and a locally free sheaf $E$ on $X$, let $X_{n}$ be the corresponding geometric fibre, and $E_{n}$ the sheaf induced on $X_{n}$. If $E_{1}, \ldots, E_{m}$ are locally free sheaves on $X$, and if $i_{1}+\cdots+i_{m}=r$, then the degree of the zero-cycle $c_{i_{1}}\left(E_{1 \eta}\right) \cdots$ $c_{i_{m}}\left(E_{m \eta}\right)$ is independent of the choice of $\eta$.

(3) The results of (1) and (2) are used to study the behavior under specialization of a closed subvariety $V^{\prime} \subset P^{2 r-1}$ which is the image under generic projection of a nonsingular $V^{r} \subset P^{n}$.
\end{abstract}

1. Introduction. Let $V^{r}$ be a nonsingular $r$-dimensional projective variety over an algebraically closed field $k$. If $V$ is projected generically onto $V^{\prime} \subset \boldsymbol{P}^{2 r-1}$, then $V^{\prime}$ has a singular curve with finitely many points of a type known as pinch points (cf. §5). Suppose that $\operatorname{char}(k)=0$ and that $V$ can be specialized (along with its projective embedding) to a nonsingular variety $V_{1}$, defined over $k_{1}$, which can be projected generically onto $V_{1}^{\prime} \subset \boldsymbol{P}^{2 r-1}$. We can ask whether $V_{1}^{\prime}$ has the same number of pinch points as $V^{\prime}$. The answer is "yes" if char $\left(k_{1}\right) \neq 2$; if $\operatorname{char}\left(k_{1}\right)=2$, then $V_{1}^{\prime}$ has half as many pinch points as $V^{\prime}$.

In this paper we develop some techniques which enable one to answer this and other enumerative questions of a similar nature. In $\S 2$, we prove a result which says roughly that a Chern polynomial of weight $r$ is constant in a connected family of nonsingular projective varieties of dimension $r$ (cf. Theorem 1). In $\S 3$ we recall the definition of the dependency cycle of a set of sections of a locally free sheaf on $V$. Mattuck [6] has shown how to express the rational equivalence class of this cycle as a Chern class of $E$. We express the multiplicities of its components as the

Received by the editors May 17, 1971.

AMS 1970 subject classifications. Primary 14C15, 14N05, 14N10.

Key words and phrases. Projective algebraic variety, generic projection, pinch point, rational equivalence ring, Chern class, locally free sheaf, Fitting ideal, singular cycle, noetherian scheme, smooth projective morphism, geometric fibre, specialization.

(1) Partially supported by NSF grant GP 20550.

Copyright (1) 1972, American Mathematical Society 
lengths of certain Fitting ideals (cf. Proposition 4). In the case that the set of sections is part of a Serre sequence, these results follow from Theorem 2.7 of [3]. In $\S 4$, we define, under suitable assumptions, the singular cycles $S_{i}$ of a morphism $f: V^{r} \rightarrow W^{m}$ of nonsingular varieties $(m \geqq r)$. Intuitively, Supp $\left(S_{i}\right)$ is the set of points where the kernel of the tangent map has dimension $\geqq i$. Our definition is stated in a form which gives immediately the multiplicities of the components of $S_{i}$. Let $V \subset \boldsymbol{P}^{n}$, and let $\pi: V \rightarrow \boldsymbol{P}^{m}$ be induced by generic projection. (See Lemma 3 for the meaning of "generic" in this context.) Theorem 2 says that the rational equivalence class of $S_{1}(\pi)$ is $c_{m-r+1}(N \otimes \mathcal{O}(-1))$, where $N$ is the normal bundle of $V$ in $\boldsymbol{P}^{n}$. Finally, $\S 5$ gives the application of the results of $\S \S 2,3$, and 4 to the problem stated in the first paragraph. We also give a concrete example to illustrate our result.

We will deal with Chern classes constructed in the rational equivalence ring $\mathscr{A}(V)$. Our references for this topic are Grothendieck's appendix to the BorelSerre paper [4], and Séminaire Chevalley 1958, "Anneaux de Chow et applications." As usual, Grothendieck's Eléments de géométrie algébrique is denoted EGA.

I would like to thank the referee, who suggested ways to clarify several obscure points.

2. Chern classes and algebraic families. Let $f: X \rightarrow S$ be a smooth projective morphism of noetherian schemes. Assume that $S$ is connected and that all fibres of $f$ are absolutely connected. In particular, $f$ is flat, and the fibre $X_{s}=f^{-1}(s)$ is an absolutely nonsingular irreducible projective variety over $\boldsymbol{k}(s)$ for all $s \in S$, where $\boldsymbol{k}(s)$ is the residue field of $\mathcal{O}_{S, s}$.

Let $E_{1}, \ldots, E_{m}$ be locally free sheaves on $X$. For each geometric point $\eta: \operatorname{Spec}(k) \rightarrow S$ ( $k$ is an algebraically closed field), let $X_{\eta}$ be the corresponding geometric fibre, and let $E_{1 \eta}, \ldots, E_{m \eta}$ be the sheaves induced on $X_{n}$ by $E_{1}, \ldots, E_{m}$. We will consider the Chern classes $c_{i}\left(E_{j n}\right)$, which are elements of $\mathscr{A}\left(X_{\eta}\right)$, the Chow ring of $X_{n}$. For a rational equivalence class, $z$, of zero-cycles on $X_{\eta}$, we will denote by $\operatorname{deg}_{\eta}(z)$ the degree of $z$. Finally, we note that the dimension of $X_{\eta}$ is independent of $\eta$.

THEOREM 1. Let $f: X \rightarrow S$ and $E_{1}, \ldots, E_{m}$ be as above. If $i_{1}, \ldots, i_{m}$ is a sequence of positive integers such that $i_{1}+\cdots+i_{m}=\operatorname{dim}\left(X_{\eta}\right)$, and $i_{j} \leqq \operatorname{rk}\left(E_{j}\right)$, for $j=1, \ldots$, $m$, then the value of

$$
\operatorname{deg}_{\eta}\left(c_{i_{1}}\left(E_{1 \eta}\right) \cdots c_{i_{m}}\left(E_{m \eta}\right)\right)
$$

is independent of the choice of the geometric point, $\eta$, of $S$.

REMARK. The $E_{i}$ need not be distinct.

Proof. We first consider the case where the $E_{j}$ are all invertible, so that $c_{i}\left(E_{j n}\right)=0$ for $i>1$. Thus let $r=\operatorname{dim}\left(X_{\eta}\right)$, and let $L_{1}, \ldots, L_{r}$ be invertible sheaves on $X$ (not necessarily distinct). Then

$$
\operatorname{deg}\left(c_{1}\left(L_{1 \eta}\right) \cdots c_{1}\left(L_{r \eta}\right)\right)=\left(L_{1 \eta} \cdots L_{r \eta}\right)
$$


which is the intersection number, computed on $X_{n}$. Using the techniques of [7, Lecture 12], one shows

$$
\begin{aligned}
& \left(L_{1 \eta} \cdots L_{r \eta}\right) \\
& \quad=\chi\left(\mathcal{O}_{X_{\eta}}\right)-\sum_{i=1}^{r} \chi\left(L_{i \eta}^{-1}\right)+\sum_{i<j} \chi\left(L_{i \eta}^{-1} \otimes L_{j \eta}^{-1}\right)-\cdots+(-1)^{r} \chi\left(L_{1 \eta}^{-1} \otimes \cdots \otimes L_{r \eta}^{-1}\right) .
\end{aligned}
$$

(By induction on the dimension, one shows that our expression is correct when $L_{1} \cong \mathcal{O}_{V}(D)$, with $D$ very ample, and is linear in each variable.)

The fact that this intersection number is independent of $\eta$ is a consequence of the following lemma.

Lemma 1. Let $f: X \rightarrow S$ be a projective morphism, where $S$ is noetherian, and let $E$ be a coherent sheaf on $X$ which is flat over $S$. For a field, $k$, and a $k$-valued point $\eta: \operatorname{Spec}(k) \rightarrow S$, let

$$
\chi(E, \eta)=\sum_{i \geqq 0}(-1)^{i} \operatorname{dim}_{k} H^{i}\left(X_{\eta}, E_{\eta}\right),
$$

where $E_{\eta}$ is the sheaf induced on the fibre $X_{\eta}$. If $S$ is connected, then $\chi(E, \eta)$ is independent of the choice of $k$ and $\eta$.

This lemma is a consequence of EGA III.7.9.11; cf. also 7.7.4, 7.7.12(i), and 7.9.3 of the same chapter.

We will now reduce the general case to the case just considered. We claim that there is a smooth projective morphism $g: Y \rightarrow X$ with connected fibres such that

(1) For each $j, g^{*} E_{j}$ has a filtration of locally free subsheaves

$$
E_{j 1} \subset E_{j 2} \subset \cdots \subset E_{j, p_{j}}=g^{*} E_{j},
$$

where $p_{j}=\mathrm{rk}\left(E_{j}\right)$, such that the quotients $L_{j v}=E_{j v} / E_{j, v-1}$ are invertible.

(2) There are invertible sheaves, $\Lambda_{1}, \ldots ; \Lambda_{s}$, on $Y$ and positive integers, $d_{1}, \ldots, d_{s}$, such that

( $\alpha) d_{1}+\cdots+d_{s}=d=$ dimension of any fibre of $g$,

( $\beta) g_{n *}\left(c_{1}\left(\Lambda_{1 \eta}\right)^{d_{1}} \cdots c_{1}\left(\Lambda_{s \eta}\right)^{d_{s}}\right)=1_{X_{\eta}}$,

for all geometric points $\eta$ : Spec $(k) \rightarrow S$, where $g_{\eta}$ is obtained by base extension.

We will first use (1) and (2) to achieve the desired reduction. From (1) we have

$$
c\left(\left(g^{*} E_{j}\right)_{\eta}\right)=\prod_{v}\left(1+c_{1}\left(L_{j v \eta}\right)\right) .
$$

Next, let $z \in \mathscr{A}\left(X_{\eta}\right)$, and set $y_{\eta}=\left(c_{1}\left(\Lambda_{1 \eta}\right)^{d_{1}} \cdots c_{1}\left(\Lambda_{s \eta}\right)^{d_{s}}\right)$. Using the projection formula (cf. [1, p. 3-17]), and (2), we obtain

$$
z=z \cdot 1_{X_{\eta}}=z \cdot g_{n *}\left(y_{\eta}\right)=g_{n *}\left(g_{\eta}^{*}(z) \cdot y_{\eta}\right) \text {. }
$$

In particular, this implies that

$$
\operatorname{deg}_{n}(z)=\operatorname{deg}_{\eta}\left(g_{n}^{*}(z) \cdot y_{n}\right)
$$


if $z$ is the class of a zero-cycle on $X_{n}$. Note that the expression on the right is the degree of a zero-cycle on $Y_{n}$.

We apply $(* *)$ in the case $z=c_{i_{1}}\left(E_{1 \eta}\right) \cdots c_{i_{m}}\left(E_{m \eta}\right)$ and use (*) to express $g_{\eta}^{*}(z) \cdot y_{n}$ as a polynomial of degree $r+d$ in the $c_{1}\left(\Lambda_{i \eta}\right)$ and the $c_{1}\left(L_{j v n}\right)$. In this way, we reduce the question to the case of the theorem already proved.

We now prove the existence of $Y$. In the case $m=1$, write $E=E_{1}$, and take $Y=$ Flag $(E)$, the flag bundle of $E$ over $X$ (cf. [1, pp. 4-18 and 4-19.]). Thus, if $p=\mathrm{rk}(E)$, we have a sequence of morphisms

$$
Y=P_{p-1} \rightarrow P_{p-2} \rightarrow \cdots \rightarrow P_{1} \rightarrow P_{0}=X,
$$

such that $P_{1}=\boldsymbol{P}(E)$, and $P_{i+1}=\boldsymbol{P}\left(F_{i}\right)$, for $i \geqq 1, F_{i}$ being defined by the exactness of $0 \rightarrow F_{i} \rightarrow q_{i}^{*}\left(F_{i-1}\right) \rightarrow \mathcal{O}_{P_{i}}(1) \rightarrow 0$, where $q_{i}: P_{i} \rightarrow P_{i-1}$, and $F_{0}=E$. Now, it is known that

$$
\begin{aligned}
q_{i n *}\left(\left(c_{1}\left(\mathcal{O}_{P_{i} \eta *}(1)\right)_{\eta}\right)^{e}\right) & =0, & & e<p-i, \\
& =1, & & e=p-i
\end{aligned}
$$

(cf. [1, p. 4-13]). Letting $g_{i}: Y \rightarrow P_{i}$ be the composition $P_{p-1} \rightarrow \cdots \rightarrow P_{i}$, we set $\Lambda_{i}=g_{i}^{*}\left(\mathcal{O}_{P_{i}}(1)\right)$ and $d_{i}=p-i$. Writing $g=g_{0}$ and using $(* * *)$ and the projection formula repeatedly, we find

$$
g_{n *}\left(c_{1}\left(\Lambda_{1 \eta}\right)^{p-1} \cdots c_{1}\left(\Lambda_{p-1, \eta}\right)\right)=1_{X_{\eta}} .
$$

In the case $m>1$, we proceed by induction on $m$. Suppose that $q: Z \rightarrow X$ and the invertible sheaves $\Lambda_{1}, \ldots, \Lambda_{s}$ on $Z$ have properties (1) and (2) relative to $E_{1}, \ldots$, $E_{m-1}$. Let $Y=$ Flag $\left(q^{*} E_{m}\right)$, and let $r: Y \rightarrow Z$. We pull back $\Lambda_{1}, \ldots, \Lambda_{s}$ to $r^{*} \Lambda_{1}, \ldots$, $r^{*} \Lambda_{s}$ on $Y$ and form $p-1$ other invertible sheaves on $Y$ by the process used in the case $m=1$, where $p=\mathrm{rk}\left(E_{m}\right)$. Setting $g=q \circ r$, one uses the projection formula and the fact that $g_{n *}=q_{n *} \circ r_{n *}$ to check that the sheaves constructed on $Y$ have property (2).

3. Dependency cycles. In this section, $V$ will be a nonsingular quasi-projective variety defined over an algebraically closed field $k$, and $E$ will be a locally free sheaf on $V$, of $\operatorname{rank} p \leqq \operatorname{dim}(V)$. We will recall the definition of the dependency cycle of a set of sections of $E$ (cf. Mattuck [6, §4]), and we will give an expression for the multiplicities of the components of this cycle.

Let $\hat{E}=\boldsymbol{P}\left(\mathcal{O}_{V} \oplus E^{*}\right)$ (cf. EGA II, §4), where $E^{*}=\mathscr{H}$ om $\left(E, \mathcal{O}_{V}\right)$, and let $\pi: \hat{E} \rightarrow V$ be the natural projection. Let $\sigma_{0}: V \rightarrow \hat{E}$ correspond to the surjection $\mathcal{O}_{V} \oplus E^{*} \rightarrow \mathcal{O}_{V}$ which restricts to the identity on $\mathcal{O}_{V}$ and to the zero map on $E^{*}$. Thus $V \cong \sigma_{0}(V)$. Let $s \in \Gamma(V, E)$, and let $\sigma: V \rightarrow \hat{E}$ correspond to the surjection $\mathcal{O}_{V} \oplus E^{*} \rightarrow \mathcal{O}_{V}$ which restricts to the identity on $\mathcal{O}_{V}$ and to the map dual to $s: \mathcal{O}_{V} \rightarrow E$ on $E^{*}$. Under the assumption that $\sigma^{-1}\left(\sigma_{0}(V)\right)=\{x \in V \mid s(x)=0\}$ is of pure codimension $p$ in $V$, we say that the cycle of zeros of $s$ is defined; this cycle is defined to be $\sigma^{*}\left(\sigma_{0}(V)\right)$ and is denoted $s^{*}(0)$. 
Let us write $s^{*}(0)=\sum \mu_{Z} Z$; the sum ranges over the irreducible components of $\sigma^{-1}\left(\sigma_{0}(X)\right)$. For a fixed $Z$, let $x$ be the generic point of $Z$, and let $U$ be a neighborhood of $x$ on which $E$ is free. On $U$, we can write $s=\sum_{i=1}^{p} f_{i} e_{i}$, where $f_{i} \in \Gamma\left(U, \mathcal{O}_{V}\right)$, and $\left\{e_{1}, \ldots, e_{p}\right\}$ is a basis of $\Gamma(U, E)$.

Proposition 1. With the above notation, $\mu_{Z}=\ell_{A}\left(A /\left(f_{1}, \ldots, f_{p}\right)\right)$, where $A=\mathcal{O}_{V, x}$, and $\ell_{A}(M)$ denotes the length of the A-module $M$.

Proof. Since $\sigma_{0}(V)$ and $\sigma(V)$ are locally complete intersections on $\hat{E}$, one can check that $\ell_{A}\left(A /\left(f_{1}, \ldots, f_{p}\right)\right)$ is the multiplicity of the corresponding component of $\sigma_{0}(V) \cdot \sigma(V)$. Q.E.D.

Proposition 2. If $s \in \Gamma(V, E)$ is such that $s^{*}(0)$ is defined, then $s^{*}(0)=c_{p}(E)$ in $\mathscr{A}(V)$.

The proof can be modeled directly after one given by Grothendieck [4, Theorem 2].

Let $q \leqq p$, and let $s_{1}, \ldots, s_{q} \in \Gamma(V, E)$. Now, $s_{1}, \ldots, s_{q}$ define a map of $\mathcal{O}_{V^{-}}$ modules, $\phi: \mathcal{O}_{V}^{q} \rightarrow E$; by duality we get $\phi^{*}: E^{*} \rightarrow \mathcal{O}_{V}^{q}$. Let $P=V \times \boldsymbol{P}^{q-1}$, and let $\pi: P \rightarrow V$ and $\rho: P \rightarrow P^{q-1}$ be the two projections. Then $\phi^{*}$ gives rise to a map of $\mathcal{O}_{P}$-modules: $\pi^{*} E^{*} \rightarrow \rho^{*} \mathcal{O}(1)$. Tensoring with $\mathcal{O}(-1)$ and dualizing, we obtain $s \in \Gamma\left(P, \pi^{*} E \otimes \mathcal{O}(1)\right)$. We will make two assumptions:

(1) $s^{*}(0)$ is defined;

(2) $\pi$ sends every irreducible component of $s^{*}(0)$ to a subvariety of codimension $p-q+1$ in $V$.

When these assumptions are satisfied, we define the dependency cycle, $D(\Sigma)$ of the set $\Sigma=\left\{s_{1}, \ldots, s_{q}\right\}$ to be $\pi_{*}\left(s^{*}(0)\right)$.

Proposition 3. Let $\Sigma \subset \Gamma(V, E)$ and $s \in \Gamma\left(P, \pi^{*} E \otimes \mathcal{O}(1)\right)$ be as above. If $D(\Sigma)$ is defined, then $D(\Sigma)=c_{p-q+1}(E)$ in $\mathscr{A}(V)$.

For a proof, see Mattuck [6, Theorem 2].

We will now give a local description of the section $s \in \Gamma\left(P, \pi^{*} E \otimes \mathcal{O}(1)\right)$. Let $U=\operatorname{Spec}(A) \subset V$ be an affine open set on which $E$ is free, and let $\left\{e_{1}, \ldots, e_{p}\right\}$ be a basis of $\Gamma(U, E)$. Thus, $s_{i}=\sum_{j=1}^{p} f_{i j} e_{j}$, where $f_{i j} \in \Gamma\left(U, \mathcal{O}_{V}\right)$, for $i=1, \ldots, q$. Let $T_{1}, \ldots, T_{q}$ be a basis of $\Gamma\left(\pi^{-1} U, \mathcal{O}(1)\right)$, and let $U_{m}$ be the open subset where $T_{m} \neq 0$, for $1 \leqq m \leqq q$. It is easy to verify:

$$
s \mid U_{m}=\left(\sum_{i=1}^{q} \sum_{j=1}^{p}\left(t_{i} / t_{m}\right) f_{i j} e_{j}\right) \otimes T_{m},
$$

where $T_{i} \mid U_{m}=\left(t_{i} / t_{m}\right) \cdot\left(T_{m} \mid U_{m}\right)$. This implies

$$
s \mid\left(\pi^{-1} U\right)=\sum_{i, j} f_{i j}\left(e_{j} \otimes T_{i}\right) .
$$

Relation $(*)$ also implies that $x \in \operatorname{Supp}(D(\Sigma))$ iff $s_{1}, \ldots, s_{q}$ become dependent in $E \otimes \boldsymbol{k}(x)$. 
Assume that $D(\Sigma)$ is defined, and let $D(\Sigma)=\sum \mu_{Z} \cdot Z$. For a fixed $Z$, let $x$ be a generic point of $Z$, and choose $U \subset V$, as above, so that $x \in U$.

Proposition 4. With the above notation and assumptions, $\mu_{Z}=\ell_{A}(A / I)$, where $A=\mathcal{O}_{V, x}$, and $I$ is the ideal in $A$ generated by the $q \times q$ minors of the $q \times p$ matrix $\left(f_{i j}\right)$. Thus, $I$ is the 0 th Fitting ideal of Coker $\left(\phi_{x}\right)$, where $\phi: \mathcal{O}_{V}^{q} \rightarrow E$ is defined by $s_{1}, \ldots, s_{q}$.

Proof. Every $q \times q$ minor of the matrix $\left(f_{i j}\right)$ vanishes along $W=Z \cap U$. Assumption (2) implies that some $(q-1) \times(q-1)$ minor of $\left(f_{i j}\right)$ is nonzero at $x$. We may assume that this minor is nonzero at all points of $U$. Thus, some subset of $\Sigma$, say $\left\{s_{1}, \ldots, s_{q-1}\right\}$, is a subset of a basis of $\Gamma(U, E)$.

Let $J \subset \Gamma\left(U, \mathcal{O}_{V}\right)$ be generated by the $q \times q$ minors of the matrix $\left(f_{i j}\right)$. Since $E \mid U$ is free, $J$ is the $(p-q)$ th Fitting ideal of Coker $\left(\Gamma\left(U, \mathcal{O}_{V}\right)^{q} \rightarrow \Gamma(U, E)\right)$. Hence $J$ is independent of the choice of basis of $\Gamma(U, E)$ (cf. Fitting [2, Hauptsatz]), and we may assume that $e_{i}=s_{i}$, for $i=1, \ldots, q-1$. Therefore $J$ is generated by the $p-q+1$ elements $f_{q q}, \ldots, f_{q p}$. Moreover, the relation $(* *)$ becomes

$$
s \mid\left(\pi^{-1} U\right)=\sum_{j=1}^{q-1}\left(e_{j} \otimes T_{j}+f_{q j}\left(e_{j} \otimes T_{q}\right)\right)+\sum_{j=q}^{p} f_{q j}\left(e_{j} \otimes T_{q}\right) .
$$

Thus, Supp $\left(s^{*}(0) \cap U\right) \subset U_{q}$. Now, $(*)$ becomes

$$
s \mid U_{q}=\sum_{j=1}^{q-1}\left(t_{j}+f_{q j}\right)\left(e_{j} \otimes T_{q}\right)+\sum_{j=q}^{p} f_{q j}\left(e_{j} \otimes T_{q}\right),
$$

where we have set $t_{q}=1$. Let $Z^{\prime}$ be the unique component of $s^{*}(0)$ lying above $Z$, let $y$ be the generic point of $Z^{\prime}$, and let $B=\mathcal{O}_{\hat{E}, y}$. If $\mu_{Z^{\prime}}$ is the multiplicity of $Z^{\prime}$ in $s^{*}(0)$, Proposition 1 implies that $\mu_{Z^{\prime}}=\ell_{B}\left(B / I^{*}\right)$, where

$$
I^{*}=\left(t_{1}+f_{q 1}, \ldots, t_{q-1}+f_{q, q-1}, f_{q q}, \ldots, f_{q p}\right) B .
$$

Further, we have isomorphisms

$$
B / I^{*} \cong(A / I)\left[T_{1}, \ldots, T_{q-1}\right] /\left(T_{1}+\bar{f}_{q 1}, \ldots, T_{q-1}+\bar{f}_{q, q-1}\right) \cong A / I .
$$

This implies that (i) $A$ and $B$ have the same residue field, and (ii) $\ell_{A}(A / I)=\ell_{B}\left(B / I^{*}\right)$. Now, (i) implies that $\pi_{*}\left(Z^{\prime}\right)=Z$. Using (ii), we obtain $\mu_{Z}=\ell_{A}(A / I)$. Q.E.D.

4. The cycles $S_{i}$. Let $f: V^{r} \rightarrow W^{m}$ be a morphism of nonsingular varieties over the algebraically closed field $k$, where $m \geqq r$. Let $\Omega_{V / W}^{1}$ be the sheaf of relative differentials, and let $S_{i} \subset V$ be the closed subset $\left\{x \mid \operatorname{dim}_{\boldsymbol{k}(x)}\left(\Omega_{V / W}^{1} \otimes k(x)\right) \geqq i\right\}$, for each $i \geqq 1$. We will say that $S_{i}$ has the proper codimension iff every irreducible component has codimension $i(m-r+i)$. If $S_{i}$ has the proper codimension, we define the cycle $S_{i}$ by

$$
S_{i}=\sum v_{Z} \cdot Z
$$


the sum ranges over all components, and $v_{Z}=\ell_{\mathcal{C}_{x}}\left(\mathcal{O}_{x} / J\right)$, where $x$ is the generic point of $Z$, and $J$ is the $(i-1)$ st Fitting ideal of $\left(\Omega_{V / W}^{1}\right)_{x}$. We must check that $\nu_{Z}$ is finite. We have an exact sequence of $\mathcal{O}_{x}$-modules

$$
\Omega_{\mathscr{C}_{y} / k}^{1} \otimes \mathcal{O}_{x} \stackrel{\phi}{\longrightarrow} \Omega_{\mathcal{O}_{x} / k}^{1} \longrightarrow \Omega_{\mathscr{C}_{x} / \mathcal{C}_{y}}^{1} \longrightarrow 0
$$

where $y=f(x)$. The first two terms in this sequence are free of rank $m$ and $r$ respectively. If we choose suitable bases for these modules, then $\phi$ is described by an $m \times r$ matrix, and $J$ is generated by its minors of rank $r-i+1$. Since $Z$ is the only component of $S_{i}$ containing $x$, the maximal ideal $\mathfrak{m}_{x}$ is the only associated prime of $J$. Therefore $\nu_{z}$ is finite. We will call the cycles $S_{i}$ the singular cycles of $f$.

Lemma 2. Suppose that $S_{1}$ is of the proper codimension. Let $Z$ be a component of $S_{1}$, with $Z \notin S_{2}$, and with generic point $x$. Then $\nu_{Z}=\ell_{\mathcal{O}_{x}}\left(\left(\Omega_{V / W}^{1}\right)_{x}\right)$.

Proof. Since $\left(\Omega_{V / W}^{1}\right)_{x}$ is generated by one element, $\left(\Omega_{V / W}^{1}\right)_{x} \cong \mathcal{O}_{x} / J$, where $J$ is the 0th Fitting ideal. Q.E.D.

If $x$ is a closed point of $V$, then $x \in S_{i}$ iff $\operatorname{dim}_{k}\left(\mathfrak{m}_{x} /\left(\mathfrak{m}_{y} \mathcal{O}_{x}+\mathfrak{m}_{x}^{2}\right)\right) \geqq i$, where $y=f(x)$, and $\mathfrak{m}_{x}$ and $\mathfrak{m}_{y}$ are the maximal ideals of $\mathcal{O}_{x}$ and $\mathcal{O}_{y}$. In particular, suppose that $V \subset \boldsymbol{P}^{n}$ and that $f=\pi: V \rightarrow \boldsymbol{P}^{m}$ is induced by projection from an $(n-m-1)-$ subspace $L \subset \boldsymbol{P}^{n}$ such that $L \cap V=\varnothing$. For a closed point $x \in V$, it follows that $x \in S_{i}$ iff $\operatorname{dim}\left(L \cap t_{V, x}\right) \geqq i-1$, where $t_{V, x}$ is the $r$-subspace of $\boldsymbol{P}^{n}$ tangent to $V$ at $x$. (This can be checked using the techniques of the proof of Proposition 3 of [8].)

Lemma 3. Let $r \leqq m<n$, and let $V^{r} \subset \boldsymbol{P}^{n}$ be nonsingular. Then there is a dense open subset of the Grassmann variety $G=G(n, n-m-1)$ consisting of linear subspaces $L \subset \boldsymbol{P}^{n}$ such that $L \cap V=\varnothing$, and $S_{i}(\pi)$ is purely of codimension $i(m-r+i)$ for all $i$, where $\pi: V \rightarrow \boldsymbol{P}^{m}$ is induced by projection from $L$.

Proof. For each $i$, consider the correspondence $Z_{i} \subset V \times G$ consisting of all $(x, L)$ such that $\operatorname{dim}\left(L \cap t_{V, x}\right) \geqq i-1$. By a counting of constants which uses standard facts about Schubert cycles on $G$ (cf. [5, Chapter XIV, §2]), one finds that $\operatorname{dim}\left(Z_{i}\right)=\operatorname{dim}(G)+r-i(m-r+i)$. Q.E.D.

Henceforth we will fix an $(n-m-1)$-subspace $L \subset P=\boldsymbol{P}^{n}$, such that $L \cap V=\varnothing$, and $S_{i}=S_{i}(\pi)$ is of the proper codimension for all $i$, where $\pi: V \rightarrow \boldsymbol{P}^{m}$ is induced by projection from $L$. We will also fix a basis, $\left\{T_{0}, \ldots, T_{n}\right\}$ of $\Gamma(P, \mathcal{O}(1))$, such that $L$ is given by $T_{0}=\cdots=T_{m}=0$.

TheOREM 2. Let $V, L$, and $\pi$ be as above. Then

$$
S_{1}=c_{m-r+1}\left(N \otimes \mathcal{O}_{V}(-1)\right) \text { in } \mathscr{A}(V),
$$

where $N$ is the normal bundle of $V$ in $\boldsymbol{P}^{n}$, viz., $N=\left(I / I^{2}\right)^{*}=\mathscr{H}_{o m_{\mathcal{O}_{V}}}\left(I / I^{2}, \mathcal{O}_{V}\right)$, where $I=\operatorname{Ker}\left(\mathcal{O}_{P} \rightarrow \mathcal{O}_{V}\right)$.

Proof. For $j=0, \ldots, n$, let $T_{j}$ be as above, and let $U_{j} \subset P$ be the open set $\left\{x \mid T_{j}(x) \neq 0\right\}$. Choose $t_{0}=1, t_{1}, \ldots, t_{n} \in k(P)$ such that $T_{i}=\left(t_{i} / t_{j}\right) T_{j}$ on $U_{j}$. Thus, 
$\Gamma\left(U_{j}, \mathcal{O}_{P}\right)$ is the polynomial ring $k\left[t_{0} / t_{j}, \ldots, t_{n} / t_{j}\right]$. For $0 \leqq i \leqq n$ and $i \neq j$, let $D_{i j}$ be the derivation of $\Gamma\left(U_{j}, \mathcal{O}_{P}\right)$ given by $D_{i j}\left(t_{v} / t_{j}\right)=\delta_{i v}$ (Kronecker delta), and let $D_{j j}=-\sum_{i \neq j}\left(t_{i} / t_{j}\right) D_{i j}$. We can extend these derivations to derivations of the function field $k(P)=k\left(t_{1}, \ldots, t_{n}\right)$ and check that $D_{i j}=\left(t_{j} / t_{h}\right) D_{i h}$ for all $i, j, h$.

For each $(i, j), D_{i j}$ induces an element $\bar{D}_{i j} \in \Gamma\left(U_{j},\left(I / I^{2}\right)^{*}\right)$. We define

$$
s_{i j}=\bar{D}_{i j} \otimes T_{j}^{-1} \in \Gamma\left(U_{j}, N \otimes \mathcal{O}(-1)\right) .
$$

Since $\bar{D}_{i j}\left|U_{j} \cap U_{h}=\left(t_{j} / t_{h}\right) \bar{D}_{i h}\right| U_{j} \cap U_{h}$, the various $s_{i j}$ (for each $i$ ) fit together to give sections $s_{0}, \ldots, s_{n} \in \Gamma(V, N \otimes \mathcal{O}(-1))$.

Let $x$ be any closed point of $V$. Since $L \cap V=\varnothing$, we have $T_{q}(x) \neq 0$ for some $q \leqq m$. We may assume $q=0$; thus $x \in U_{0}$. Let $g_{1}, \ldots, g_{n-r}$ generate $I$ in a neighborhood of $x$, and let $\sigma_{i}: I / I^{2} \rightarrow \mathcal{O}_{V}$ be given locally by $\sigma_{i}\left(\left[g_{j}\right]\right)=\delta_{i j}$. With these notations, we find that

$$
s_{i}=\sum_{j=1}^{n-r}\left(\partial g_{j} / \partial t_{i}\right)\left(\sigma_{j} \otimes T_{0}^{-1}\right)
$$

in some neighborhood of $x$. (The $g_{j}$ are polynomials in $t_{1}, \ldots, t_{n}$.) In particular, it follows that $s_{m+1}, \ldots, s_{n}$ become dependent in $(N \otimes \mathcal{O}(-1)) \otimes \boldsymbol{k}(x)$ iff $x \in S_{1}$. To see this, we note that $L \cap t_{V, x} \neq \varnothing$ iff there are elements $b_{m+1}, \ldots, b_{n} \in k$, not all zero, such that $\sum_{j=m+1}^{n} b_{j} \cdot\left(\partial g_{i} / \partial t_{j}\right)(x)=0$, for $i=1, \ldots, n-r$. Since $S_{1}$ is purely of codimension $m-r+1=(n-r)-(n-m)+1$, it follows that $D(\Sigma)$ is defined $\left(\Sigma=\left\{s_{m+1}, \ldots, s_{n}\right\}\right)$, and $\operatorname{Supp}(D(\Sigma))=\operatorname{Supp}\left(S_{1}\right)$. Proposition 4 implies that $D(\Sigma)=\sum \mu_{Z} Z$, where $\mu_{Z}=\ell_{\mathcal{C}_{x}}\left(\mathcal{O}_{x} / J^{\prime}\right)$, with $x$ the generic point of $Z$, and $J^{\prime}$ the ideal in $\mathcal{O}_{x}$ generated by the minors of order $(n-m-1)$ of the $(n-r) \times(n-m-1)$ matrix $\left(\partial g_{i} / \partial t_{j}\right)_{1 \leqq i \leqq n-r, m+1 \leqq j \leqq n}$. Finally, Proposition 3 implies that $D(\Sigma)=$ $c_{m-r+1}\left(N \otimes \mathcal{O}_{V}(-1)\right)$ in $\mathscr{A}(V)$.

Since $S_{1}$ and $D(\Sigma)$ have the same irreducible components, it will follow that $S_{1}=D(\Sigma)$ if we can show that the ideal $J^{\prime}$ defined above is the 0th Fitting ideal of $\Omega_{\mathscr{O}_{x} / \mathbb{C}_{y}}^{1}(y=\pi(x))$. This will complete the proof. Thus, let $U=\boldsymbol{P}^{n}-L=U_{0} \cup \cdots$ $\cup U_{m}$. The projection $\pi: \boldsymbol{P}^{n}-L \rightarrow \boldsymbol{P}^{m}$ has the property that $\pi \mid U_{i}$ looks like the projection $\boldsymbol{A}^{m} \times \boldsymbol{A}^{n-m} \rightarrow \boldsymbol{A}^{m}$. Hence in the exact sequence of $\mathcal{O}_{V}$-modules

$$
I / I^{2} \stackrel{\delta}{\longrightarrow} \Omega_{U / P^{m}}^{1} \otimes \mathcal{O}_{V} \longrightarrow \Omega_{V / P^{m}}^{1} \longrightarrow 0,
$$

the first two terms are free. Moreover, if $x \in U_{0}$, and if $g_{1}, \ldots, g_{n-r}$ generate $I_{x}$, then $\delta$ is given by

$$
\delta\left(\left[g_{i}\right]\right)=\sum_{j=m+1}^{n}\left(\partial g_{i} / \partial t_{j}\right)\left(d t_{j} \otimes 1\right),
$$

for $i=1, \ldots, n-r$. We conclude that $J^{\prime}$ is the 0 th Fitting ideal of $\Omega_{V / P^{m}}^{1}$. Q.E.D.

COROLlARY 1. With the assumptions of Theorem 2,

$$
S_{1}=\sum_{j=0}^{m-r+1}\left(\begin{array}{c}
m+1 \\
j
\end{array}\right) \gamma_{q-j} h^{j} \text { in } \mathscr{A}(V)
$$


where $h$ is the divisor class of a hyperplane section, $\left(1-\gamma_{1}+\gamma_{2}-\cdots+(-1)^{r} \gamma_{r}\right)$ $=c\left(\Omega_{V}^{1}\right)^{-1}$, and $q=m-r+1$.

Proof. With $P=\boldsymbol{P}^{n}$, let $D_{i j}$ be the derivations of $k(P)$ defined above. Let $\Theta$ $=\left(\Omega_{P}^{1}\right)^{*}$, and let $s_{0}, \ldots, s_{n} \in \Gamma\left(P, \Theta \otimes \mathcal{O}_{P}(-1)\right)$ satisfy $s_{i} \mid U_{j}=D_{i j} \otimes T_{j}^{-1}$. The $s_{i}$ give a map $\psi: \mathcal{O}_{P}^{n+1} \rightarrow \Theta \otimes \mathcal{O}_{P}(-1)$ and thus an exact sequence

$$
0 \rightarrow \Omega_{P}^{1} \rightarrow \mathcal{O}_{P}(-1)^{n+1} \rightarrow \mathcal{O}_{P} \rightarrow 0 .
$$

This gives $c\left(\Omega_{P}^{1}\right)=(1-h)^{n+1}$.

On $V$ we have an exact sequence

$$
0 \rightarrow\left(I / I^{2}\right) \rightarrow \Omega_{P}^{1} \otimes \mathcal{O}_{V} \rightarrow \Omega_{V}^{1} \rightarrow 0 .
$$

Thus $c\left(N^{*}\right)=j^{*}\left(c\left(\Omega_{P}^{1}\right)\right) \cdot c\left(\Omega_{V}^{1}\right)^{-1}$, where $j: V \rightarrow P$. The rest of the computation will be omitted.

COROLlaRy 2. If $m=r=1$, and if the curve $V \subset \boldsymbol{P}^{n}$ has genus $g$ and degree $d$, then

$$
2 g-2=-2 d+\sum_{x \in S_{1}(\pi)} \ell_{\mathcal{O}_{x}}\left(\left(\Omega_{V / P^{1}}^{1}\right)_{x}\right) .
$$

This is a special case of Hurwitz' formula for the genus change under a morphism of curves. For a proof, use the exact sequence

$$
0 \rightarrow I / I^{2} \rightarrow j^{*}\left(\Omega_{P^{n} / k}^{1}\right) \rightarrow \Omega_{V / k}^{1} \rightarrow 0
$$

(with $\left.j: V \rightarrow \boldsymbol{P}^{n}\right)$ to show that $\operatorname{deg}(N \otimes \mathcal{O}(-1))=2 g+2 d-2$. Lemma 2 shows that the summation on the right side of the formula also is $\operatorname{deg}(N \otimes \mathcal{O}(-1))$.

Generalization. It is also possible to express the cycles $S_{i}, i>1$, in terms of Chern classes. Thus let $G^{\prime}=G^{\prime}(n, n-r-1)$ be the Grassmannian which parameterizes $(n-r)$-quotients of rank $n+1$ free sheaves, and let $\Phi: \mathcal{O}_{G^{\prime}}^{n+1} \rightarrow E$ be the universal surjection. There is a morphism $u: V \rightarrow G^{\prime}$ such that $\Phi$ pulls back to $\psi: \mathcal{O}_{V}^{n+1} \rightarrow N \otimes \mathcal{O}_{V}(-1)$. The Chern classes of $E$ can be expressed in terms of Schubert cycles on $G^{\prime}$. On the other hand, it seems clear that the Schubert cycles which pull back to the cycles $S_{i}$ can be expressed in terms of the Fitting ideals of Coker $(\Phi)$. Thus, one should obtain formulas which are similar to formula (10) on p. 357 of [5].

5. Enumeration of pinch points. Let $V^{r}$ be a nonsingular projective variety over an algebraically closed field $k$. One can find a projective embedding $V \subset \boldsymbol{P}^{n}$ such that there is a finite morphism $\pi: V \rightarrow P^{2 r-1}$ induced by projection from an $(n-2 r)$-subspace $L \subset \boldsymbol{P}^{n}$ satisfying

(I) $S_{1}(\pi)$ is purely 0 -dimensional, and $\pi \mid S_{1}(\pi)$ is injective. Moreover, if $r \geqq 2$, then $\pi^{-1}(\pi(x))=\{x\}$ for all $x \in S_{1}(\pi)$.

(II) If $x \in S_{1}(\pi)$ and $y=\pi(x)$, then $\hat{\mathcal{O}}_{V, x}$ and $\hat{\mathcal{O}}_{P^{2 r-1}, y}$ can be identified with formal power series rings $B=k\left[\left[t_{1}, \ldots, t_{r}\right]\right]$ and $A=k\left[\left[t_{1}, \ldots, t_{2 r-1}\right]\right]$ so that $\pi$ induces the 
homomorphism $f: A \rightarrow B$ given by

$$
\begin{aligned}
f\left(t_{i}\right) & =t_{i} & & \text { for } i=1, \ldots, r-1, \\
f\left(t_{i}\right) & =t_{i-r+1} t_{r} & & \text { for } i=r, \ldots, 2 r-2, \\
f\left(t_{2 r-1}\right) & =t_{r}^{2}+t_{r}^{3} . & &
\end{aligned}
$$

(If $\operatorname{char}(k) \neq 2$ we can replace $t_{r}^{2}+t_{r}^{3}$ by $t_{r}^{2}$.)

In fact, if $r \geqq 2$, a suitable embedding may be found by replacing any given embedding by the embedding determined by hypersurface sections of degree $d \geqq 2$, and Theorem 3 of [8] states that if $L$ is chosen generically, then $\pi$ has the following properties which imply (I) and (II). If $V^{\prime}=\pi(V)$, then $V^{\prime}$ is birational to $V$, Sing $\left(V^{\prime}\right)$ is purely of dimension 1 , and $V^{\prime}$ has singular branches at only finitely many closed points $y \in V^{\prime}$. If $V^{\prime}$ has a singular branch at $y$, then $\hat{\mathcal{O}}_{V^{\prime}, y}$ is isomorphic to $f(A)$, where $f: A \rightarrow B$ is as above. (Recall that if $\{x\}=\pi^{-1}(y)$, then $x \in S_{1}(\pi)$ iff $y$ has a singular branch at $y$.)

If $r=1$, and $V$ is of genus $g$, one embeds $V$ by using a complete linear system of degree $\geqq 2 g+3$ and uses techniques like those used in the proof of Theorem 3 of [8] to obtain (I) and (II).

Proposition 5. Let $\pi: V \rightarrow \boldsymbol{P}^{2 r-1}$ be as above, and let $S_{1}=\sum_{x} \nu_{x} \cdot x$, where the summation extends over all points of $\operatorname{Supp}\left(\Omega_{V / P^{2 r}-1}^{1}\right)$.

If $\operatorname{char}(k) \neq 2$, then $\nu_{x}=1$ for all $x$.

If $\operatorname{char}(k)=2$, then $\nu_{x}=2$ for all $x$.

Proof. Let $x \in S_{1}(\pi)$ and $y=\pi(x)$. Since $\pi$ is finite, $\mathcal{O}_{x} \cong R_{\mathrm{m}}$, where $R$ is a semilocal ring which is a finite $\mathcal{O}_{y}$-module, and $\mathfrak{m}$ is maximal. Hence, $\hat{R} \cong R \otimes_{\mathcal{C}_{y}} \hat{\mathcal{O}}_{y}$, and $\hat{\mathcal{O}}_{x} \cong R / \mathfrak{a}$, where $\mathfrak{a}$ is generated by idempotent elements. Therefore, $\Omega^{1} \hat{\mathscr{O}}_{x} / \hat{\mathcal{O}}_{y} \cong \Omega_{\hat{O}_{x} / \mathcal{C}_{y}}^{1}$ $\otimes \hat{\mathcal{O}}_{x}$, so that the 0th Fitting ideal of $\Omega^{1} \hat{\mathscr{O}}_{x} / \hat{\mathcal{O}}_{y}$ is $I \cdot \hat{\mathcal{O}}_{x}$, where $I$ is the 0th Fitting ideal of $\Omega_{\mathscr{O}_{x} / \mathscr{C}_{y}}^{1}$. Let $A=k\left[\left[t_{1}, \ldots, t_{2 r-1}\right]\right], B=k\left[\left[t_{1}, \ldots, t_{r}\right]\right]$, and let $f: A \rightarrow B$ be given as above. Then $\ell_{\mathcal{C}_{x}}\left(\mathcal{O}_{x} / I\right)=\ell_{B}(B / J)$, where $J$ is the 0 th Fitting ideal of $\Omega_{B / A}^{1}$. We have an exact sequence of $B$-modules

$$
\hat{\Omega}_{A / k}^{1} \hat{\otimes}_{A} B \stackrel{u}{\longrightarrow} \Omega_{B / k}^{1} \longrightarrow \Omega_{B / A}^{1} \longrightarrow 0 .
$$

(Cf. EGA $0_{\mathrm{IV}}, 20.7 .17 .3$ and $0_{\mathrm{I}}$, 7.3.5.) The first two terms are free, and $u$ is given by

$$
\begin{aligned}
u\left(d t_{i} \otimes 1\right) & =d t_{i} & & \text { for } i=1, \ldots, r-1, \\
u\left(d t_{i} \otimes 1\right) & =t_{r} d t_{i-r+1}+t_{i-r+1} d t_{r} & & \text { for } i=r, \ldots, 2 r-2, \\
u\left(d t_{2 r-1} \otimes 1\right) & =\left(2 t_{r}+3 t_{r}^{2}\right) d t_{r} . & &
\end{aligned}
$$

The 0th Fitting ideal is thus $J=\left(t_{1}, \ldots, t_{r-1}, t_{r}\right)$ if char $(k) \neq 2$, and

$$
J=\left(t_{1}, \ldots, t_{r-1}, t_{r}^{2}\right)
$$

if $\operatorname{char}(k)=2$. The length of $B / J$ is 1 (resp. $=2$ ) if $\operatorname{char}(k) \neq 2$ (resp. $=2$ ). Q.E.D.

RemarK. As a consequence of Proposition 5, the number of points in $S_{1}(\pi)$ is independent of the choice of projection center, $L$, provided (I) and (II) are satisfied. 
We will now see how the number of points varies as $V$ is specialized. Thus, let $A$ be a noetherian ring, and $X$ a closed subscheme of $\boldsymbol{P}_{A}^{n}=\operatorname{Proj} A\left[T_{0}, \ldots, T_{n}\right]$; assume that $p: X \rightarrow \operatorname{Spec}(A)$ is smooth and has absolutely irreducible fibres of dimension $r$. Assume that $n \geqq 2 r-1$ and that $X$ does not meet the closed subscheme given by $T_{0}=\cdots=T_{2 r-1}=0$. We define $N$ to be the normal bundle of $X$ in $P=\boldsymbol{P}_{A}^{n}$; thus $N=\left(I / I^{2}\right)^{*}, I=\operatorname{Ker}\left(\mathscr{O}_{P} \rightarrow \mathcal{O}_{X}\right)$.

We consider geometric points $\eta: \operatorname{Spec}(k) \rightarrow \operatorname{Spec}(A)(k=\bar{k})$ such that the projection $\pi_{\eta}$ of the geometric fibre $X_{\eta} \subset \boldsymbol{P}_{k}^{n}$ from the linear subspace $T_{0}=\cdots$ $=T_{2 r-1}=0$ satisfies (I) and (II) above. Now, the bundle $N_{n}$ induced on $X_{n}$ by $N$ is just the normal bundle of $X_{n}$ in $\boldsymbol{P}_{k}^{n}$. Thus, by Theorem 2, the degree of the cycle $S_{1}\left(\pi_{\eta}\right)$ is $\operatorname{deg}\left(c_{r}\left(\left(N \otimes \mathcal{O}_{X}(-1)\right)_{\eta}\right)\right)$. By Theorem 1 , this is independent of $\eta$. Using Proposition 5, we obtain the following conclusion.

Proposition 6. Let $A$ and $X \subset \boldsymbol{P}_{A}^{n}$ be as above. Assume that $\mathrm{Spec}(A)$ is connected. For $i=1,2$, let $\eta_{i}: \operatorname{Spec}\left(k_{i}\right) \rightarrow \operatorname{Spec}(A)$ be geometric points such that the corresponding projections $\pi_{n_{i}}$ both satisfy (I) and (II). If char $\left(k_{1}\right)$ and char $\left(k_{2}\right)$ are both $\neq 2$ or both $=2$, then \# (points in $\left.S_{1}\left(\pi_{\eta_{1}}\right)\right)=\#\left(\right.$ points in $\left.S_{1}\left(\pi_{\eta_{2}}\right)\right)$. If char $\left(k_{1}\right)=2$ and char $\left(k_{2}\right) \neq 2$, then \#(points in $\left.S_{1}\left(\pi_{\eta_{1}}\right)\right)=\frac{1}{2}\left(\#\left(\right.\right.$ points in $\left.S_{1}\left(\pi_{\eta_{2}}\right)\right)$ ).

If $r \geqq 2$, we substitute \#(pinch-points of $\pi_{n_{i}}\left(X_{\eta_{i}}\right)$ ) for \#(points in $S_{1}\left(\pi_{\eta_{i}}\right)$ ) to obtain a statement about the behavior under specialization of the number of pinch-points. If $r=1$, we obtain a similar statement about the behavior under specialization of the number of ramification points of the covering $V \rightarrow \boldsymbol{P}^{1}$.

EXAMPLE. Let $V^{2} \subset \boldsymbol{P}^{5}$ be the Veronese surface, i.e. the image of $\boldsymbol{P}^{2}$ embedded by the complete linear system of conics. Explicitly, let points of $\boldsymbol{P}^{5}$ have homogeneous coordinates $\left(y_{i j}\right)$ with $0 \leqq i \leqq j \leqq 2$. Then $\left(x_{0}, x_{1}, x_{2}\right) \in \boldsymbol{P}^{2}$ is sent to the point of $\boldsymbol{P}^{5}$ with $y_{i j}=x_{i} x_{j}$. Let $\pi: V \rightarrow \boldsymbol{P}^{3}$ be induced by projection from the line $y_{01}$ $=y_{02}=y_{12}=y_{00}+y_{11}+y_{22}=0$. Thus, the composed map $\boldsymbol{P}^{2} \rightarrow \boldsymbol{P}^{3}$ sends $\left(x_{0}, x_{1}, x_{2}\right)$ to $\left(x_{1} x_{2}, x_{0} x_{2}, x_{0} x_{1}, x_{0}^{2}+x_{1}^{2}+x_{2}^{2}\right)$, and the image is the surface $V^{\prime} \subset \boldsymbol{P}^{3}$ whose equation is $t_{0}^{2} t_{1}^{2}+t_{0}^{2} t_{2}^{2}+t_{1}^{2} t_{2}^{2}-t_{0} t_{1} t_{2} t_{3}=0$. The singular locus of $V^{\prime}$ consists of the three lines $\Lambda_{0}, \Lambda_{1}$, and $\Lambda_{2}$ given respectively by $t_{1}=t_{2}=0, t_{0}=t_{2}=0$, and $t_{0}=t_{1}=0$. If char $(k) \neq 2$, then $V^{\prime}$ has 6 pinch-points, two of which lie on each of the lines $\Lambda_{i}$; if $\operatorname{char}(k)=2$, then $V^{\prime}$ has 3 pinch-points, one on each $\Lambda_{i}$.

To see this, note that $C_{i}=\pi^{-1}\left(\Lambda_{i}\right)$ is a plane conic for each $i$. The projection center meets the plane of $C_{i}$ in a point which lies on two tangent lines of $C_{i}$ if char $(k) \neq 2$, but on just one tangent line of $C_{i}$ if $\operatorname{char}(k)=2$. (The thing to note is that if char $(k)=2$, there is a point, not on the projection center, which lies on every tangent line of $C_{i}$.) It might also be noted that the plane conic provides the simplest example of the case $r=1$ of Proposition 6.

\section{REFERENCES}

1. Séminaire C. Chevalley 2e année: 1958. Anneaux de Chow et applications, Secrétariat mathématique, Paris, 1958. MR 22 \#1572.

2. H. Fitting, Die Determinantenideale eines Moduls, Jber. Deutsch. Math.-Verein. 1936, 195-228. 
3. J. Fogarty and D. S. Rim, Serre sequences and Chern classes, J. Algebra 10 (1968), 436-447. MR 39 \#2771.

4. A. Grothendieck, La théorie des classes de Chern, Bull. Soc. Math. France 86 (1958), 137-154. MR 22 \#6818.

5. W. V. D. Hodge and D. Pedoe, Methods of algebraic geometry. Vol. 2, Cambridge Univ. Press, New York, 1952. MR 13, 972.

6. A. Mattuck, Secant bundles on symmetric products, Amer. J. Math. 87 (1965), 779-797. MR $33 \# 7345$.

7. D. Mumford, Lectures on curves on an algebraic surface, Ann. of Math. Studies, no. 59, Princeton Univ. Press, Princeton, N. J., 1966. MR 35 \#187.

8. J. Roberts, Generic projections of algebraic varieties, Amer. J. Math. 93 (1971), 191-215.

Department of Mathematics, Purdue University, Lafayette, Indiana 47907 